\title{
Dense pairs of o-minimal structures
}

\author{
by \\ Lou van den Dries (Urbana, Ill.)
}

\begin{abstract}
The structure of definable sets and maps in dense elementary pairs of o-minimal expansions of ordered abelian groups is described. It turns out that a certain notion of "small definable set" plays a special role in this description.
\end{abstract}

Introduction. In a classical paper [8] A. Robinson proved the completeness of the theory of real closed fields with a predicate for a proper dense real closed subfield. Here I generalize this work of Robinson to o-minimal expansions of ordered abelian groups. My main objective, however, is to characterize definable sets and definable functions in dense elementary pairs of such structures. The results obtained in this direction are, to my knowledge, also new in the case considered by Robinson. For an extension of [8] in another direction, see Macintyre [3]. I now proceed to precise definitions and statements.

Throughout, $T$ denotes a complete o-minimal theory that extends the theory of ordered abelian groups with distinguished positive element 1 . Thus the language $L$ of $T$ extends $\{<, 0,1,+,-\}$ and $T$ has definable Skolem functions. In general, we use the same notations and conventions as in [1]. In particular, "intervals" are always open intervals $(a, b)$ with $-\infty \leq a<$ $b \leq \infty$, and we let $k, m, n$ range over $\mathbb{N}=\{0,1,2, \ldots\}$. Unless indicated otherwise, "definable" means "definable with parameters". We let $\mathcal{A}, \mathcal{B}, \mathcal{C}$, $\mathcal{D}$ (possibly with subscripts or superscripts) denote models of $T$, and $A$, $B, C, D$ their underlying sets, if it is useful to make this distinction. An elementary pair is a pair $(\mathcal{B}, \mathcal{A})$ where $\mathcal{A}$ is an elementary substructure of $\mathcal{B}$ (with $\mathcal{A}$ and $\mathcal{B}$ both models of $T$ according to our convention). A dense elementary pair (or just dense pair for simplicity) is an elementary pair $(\mathcal{B}, \mathcal{A})$ such that $A \neq B$ and $\mathcal{A}$ is dense in $\mathcal{B}$, that is, every interval in $\mathcal{B}$ contains elements of $A$. We let $T^{2}$ denote the theory whose models are exactly the elementary pairs, formulated in the language $L^{2}$ which extends

1991 Mathematics Subject Classification: 03C10, 03C35, 03C60, 06F20. 
$L$ by an extra unary predicate symbol $U$ to denote the (underlying set of the) elementary substructure. We let $T^{\mathrm{d}}$ be the theory of dense pairs, also formulated in the language $L^{2}$.

Given a dense pair $(\mathcal{B}, \mathcal{A})$ we say that a set $X \subseteq B$ is $\mathcal{A}$-small if $X$ is definable in $(\mathcal{B}, \mathcal{A})$ and $X \subseteq f\left(A^{n}\right)$ for some function $f: B^{n} \rightarrow B$ that is definable in $\mathcal{B}$. For example, let $\left(\mathbb{R}, \mathbb{R}^{\mathrm{a}}\right)$ denote the ordered field of real numbers with a predicate for the subfield $\mathbb{R}^{\mathrm{a}}$ of algebraic real numbers. Then every $\mathbb{R}^{\mathrm{a}}$-small subset of $\mathbb{R}$ is countable. The first section proves the following key fact, which is related to Macintyre's "Assumption 4" in [3]:

If $(\mathcal{B}, \mathcal{A})$ is a dense pair, then the set $B$ is not $\mathcal{A}$-small.

In Section 2 we give a back-and-forth proof of the completeness of $T^{\mathrm{d}}$, which on closer analysis leads to the following elimination of quantifiers (in a suitably extended language).

TheOREM 1. Each $L^{2}$-formula $\psi\left(y_{1}, \ldots, y_{n}\right)$ is equivalent in $T^{\mathrm{d}}$ to a boolean combination of formulas of the form

$$
\exists x_{1} \ldots \exists x_{m}\left(U\left(x_{1}\right) \& \ldots \& U\left(x_{m}\right) \& \phi(x, y)\right)
$$

where $\phi\left(x_{1}, \ldots, x_{m}, y_{1}, \ldots, y_{n}\right)$ is an L-formula.

I thank Anand Pillay for pointing out this theorem and its proof. (It replaces an embedding property and model-completeness result in an earlier version of this paper, and led to more efficient proofs of several lemmas and corollaries.)

Certain kinds of definable sets are characterized in more detail in Theorems $2-5$ below. In these results $(\mathcal{B}, \mathcal{A})$ denotes a dense pair.

THEOREM 2. Given a set $Y \subseteq A^{n}$ the following are equivalent:

(1) $Y$ is definable in $(\mathcal{B}, \mathcal{A})$;

(2) $Y=Z \cap A^{n}$ for some set $Z \subseteq B^{n}$ that is definable in $\mathcal{B}$;

(3) $Y$ is definable in the structure $\left(\mathcal{A},(A \cap(0, b))_{0<b \in B}\right)$ which expands $\mathcal{A}$ by the traces on $A$ of the intervals $(0, b)$ in $\mathcal{B}$.

It follows that the structure $\left(\mathcal{A},(A \cap(0, b))_{0<b \in B}\right)$ (and in fact its theory) is weakly o-minimal, a result that for $T=\mathrm{RCF}$ is due to MacPherson, Marker and Steinhorn [4]. Here and below RCF denotes the theory of ordered real closed fields.

Turning now our attention to definable functions, an instructive example is the function $f: \mathbb{R} \rightarrow \mathbb{R}$ defined by $f(x)=r$ if $x=r+s e$ for (necessarily unique) algebraic reals $r$ and $s$, where $e$ is the usual (transcendental) real number, while $f(x)=0$ if $x$ is not of this form. This function is definable in the dense pair $\left(\mathbb{R}, \mathbb{R}^{\mathrm{a}}\right)$, and not continuous at any point; in fact, the graph of $f$ is dense in the plane $\mathbb{R}^{2}$. This example shows that definable functions 
can be rather wild from the "graphical" point of view. Nevertheless, $f$ is the identity on $\mathbb{R}^{\mathrm{a}}$ and is constant off the $\mathbb{R}^{\mathrm{a}}$-small set $\mathbb{R}^{\mathrm{a}}+e \mathbb{R}^{\mathrm{a}}$.

The next theorem is established in Section 3 and shows that this kind of behaviour is the general rule. It also shows how definability in $(\mathcal{B}, \mathcal{A})$ is related to definability in $\mathcal{B}$ and in $\mathcal{A}$.

Theorem 3. (1) If $F: B \rightarrow B$ is definable in $(\mathcal{B}, \mathcal{A})$, then $F$ agrees off some $\mathcal{A}$-small subset of $B$ with a function $\widehat{F}: B \rightarrow B$ that is definable in $\mathcal{B}$.

(2) If $S \subseteq B$ is definable in $(\mathcal{B}, \mathcal{A})$, then there exists an $\mathcal{A}$-small set $X \subseteq B$ such that $S \backslash X=S^{\prime} \backslash X$ for some set $S^{\prime} \subseteq B$ that is definable in $\mathcal{B}$.

(3) If $f: A^{n} \rightarrow A$ is definable in $(\mathcal{B}, \mathcal{A})$, then $f$ is piecewise given by functions that are definable in $\mathcal{A}$ : there are functions $f_{1}, \ldots, f_{k}: A^{n} \rightarrow A$ definable in $\mathcal{A}$ such that for each $x \in A^{n}$ there is $i \in\{1, \ldots, k\}$ with $f(x)=$ $f_{i}(x)$.

A consequence is that if $T$ extends RCF and $|A|<|B|$, then the $\mathcal{A}$-small subsets of $B$ are exactly the subsets of $B$ that are definable in $(\mathcal{B}, \mathcal{A})$ and of cardinality $\leq|A|$, and all other subsets of $B$ definable in $(\mathcal{B}, \mathcal{A})$ have cardinality $|B|$. (Here and below $|S|$ denotes the cardinality of a set $S$.)

In Section 4 we describe one-variable definable sets and open definable sets:

Theorem 4. If $X \subseteq B$ is $\mathcal{A}$-small, then there is a partition

$$
-\infty=b_{0}<b_{1}<\ldots<b_{k}<b_{k+1}=+\infty
$$

of $B$ such that for each $i=0, \ldots, k$ either $X \cap\left(b_{i}, b_{i+1}\right)=\emptyset$, or $X \cap\left(b_{i}, b_{i+1}\right)$ as well as $\left(b_{i}, b_{i+1}\right) \backslash X$ are dense in $\left(b_{i}, b_{i+1}\right)$.

If $S \subseteq B$ is definable in $(\mathcal{B}, \mathcal{A})$, then there is a partition

$$
-\infty=b_{0}<b_{1}<\ldots<b_{k}<b_{k+1}=+\infty
$$

of $B$ such that for each $i=0, \ldots, k$ either $S \cap\left(b_{i}, b_{i+1}\right)=\emptyset$, or $\left(b_{i}, b_{i+1}\right) \subseteq S$, or $S \cap\left(b_{i}, b_{i+1}\right)$ as well as $\left(b_{i}, b_{i+1}\right) \backslash S$ are dense in $\left(b_{i}, b_{i+1}\right)$.

Hence subsets of $B$ that are definable in $(\mathcal{B}, \mathcal{A})$ and open are finite unions of intervals in $\mathcal{B}$. Similarly, discrete subsets of $B$ that are definable in $(\mathcal{B}, \mathcal{A})$ are finite. ("Discrete" means that each point of the set lies in an interval that contains no other points of the set.)

In combination with recent work by Miller and Speissegger [5] this leads to

TheOrem 5. Suppose $\mathcal{B}$ is an expansion of the ordered field of real numbers. If $S \subseteq \mathbb{R}^{n}$ is open and definable in $(\mathcal{B}, \mathcal{A})$, then $S$ is definable in $\mathcal{B}$.

For some nice consequences of this last theorem, see the end of Section 4. 
Further notations and conventions. Given a language $\mathcal{L}$ and a set $E$ we let $\mathcal{L}(E)$ be the language obtained from $\mathcal{L}$ by adding a new constant symbol $c$ for each element $c \in E$. Often $E$ is given as a subset of the underlying set of an $\mathcal{L}$-structure, and then that $\mathcal{L}$-structure will also be regarded as an $\mathcal{L}(E)$-structure in the obvious way. Given $\mathcal{L}$-structures $\mathcal{M}$ and $\mathcal{N}$ we write $\mathcal{M} \subseteq \mathcal{N}$ to indicate that $\mathcal{M}$ is a substructure of $\mathcal{N}$.

From now on we assume for convenience that $T$ admits QE and is universally axiomatizable: this extra assumption on $T$ does not affect the theorems as stated above since we can always achieve this situation by passing from $T$ to a suitable extension by definitions of $T$. A consequence of this convention is that substructures of models of $T$ are elementary substructures.

Let $\mathcal{A}$ and $\mathcal{B}$ be models of $T$ with $\mathcal{A} \subseteq \mathcal{B}$, that is, $\mathcal{A}$ is a substructure of $\mathcal{B}$. Given a set $X \subseteq B$ we let $\mathcal{A}\langle X\rangle$ denote the definable closure of $A \cup X$ in $\mathcal{B}$. Thus $\mathcal{A}\langle X\rangle$ is the underlying set of a substructure of $\mathcal{B}$, which we also denote by $\mathcal{A}\langle X\rangle$. The operation that associates with each $X \subseteq B$ the set $\mathcal{A}\langle X\rangle$ is a pregeometry on $B$ by a result of Pillay and Steinhorn [7]. In particular, we say that $X$ is independent over $\mathcal{A}$ if $\mathcal{A}\langle X\rangle \neq \mathcal{A}\langle Y\rangle$ for each proper subset $Y$ of $X$, and that $X$ is a basis of $\mathcal{B}$ over $\mathcal{A}$ if $X$ is independent over $\mathcal{A}$ and $\mathcal{A}\langle X\rangle=\mathcal{B}$. The cardinality of a basis of $\mathcal{B}$ over $\mathcal{A}$ is independent of the choice of basis and is called the rank of $\mathcal{B}$ over $\mathcal{A}$, written as $\operatorname{rk}(\mathcal{B} \mid \mathcal{A})$.

For $n>0, x, y \in B^{n}$ and nonempty $S \subseteq B^{n}$, definable in $\mathcal{B}$, we put $d(x, y):=\max \left\{\left|x_{i}-y_{i}\right|: i=1, \ldots, n\right\}$ and $d(x, S):=\inf \{d(x, s): s \in S\}$.

\section{Small sets}

Lemma 1.1. Suppose $T$ extends $R C F$. Let $\mathcal{A} \prec \mathcal{B}$, let $f: B^{n+1} \rightarrow B$ be $A$-definable in $\mathcal{B}$, and let $b \in B \backslash A$. Then there exist $a_{0}, \ldots, a_{n} \in A$ such that

$$
a_{0}+a_{1} b+\ldots+a_{n} b^{n} \notin f\left(A^{n} \times\{b\}\right) .
$$

Proof. Otherwise there is for each $a=\left(a_{0}, \ldots, a_{n}\right) \in A^{n+1}$ a tuple $\alpha \in$ $A^{n}$ such that $p(a, b)=f(\alpha, b)$, where $p(a, x)=a_{0}+a_{1} x+\ldots+a_{n} x^{n}$ for $x \in B$. Since $b \notin A$ it follows that for $a$ and $\alpha$ as above we have $p(a, x)=f(\alpha, x)$ for infinitely many $x \in A$. Since $T$ has definable Skolem functions there is an $A$-definable map $\alpha^{*}: A^{n+1} \rightarrow A^{n}$ such that for all $a \in A^{n+1}$ there is an interval $I$ in $\mathcal{A}$ with $p(a, x)=f\left(\alpha^{*}(a), x\right)$ for all $x \in I$. For dimension reasons there exist then a 1-dimensional cell $E$ in $A^{n+1}$ and a tuple $\alpha \in A^{n}$ such that $\alpha^{*}(a)=\alpha$ for all $a \in E$. Again by definability of Skolem functions there are $A$-definable functions $\beta^{*}, \gamma^{*}: E \rightarrow A$ such that if $a \in E$, then $\beta^{*}(a)<\gamma^{*}(a)$ and $p(a, x)=f(\alpha, x)$ for all $x \in\left(\beta^{*}(a), \gamma^{*}(a)\right)$. By cell decomposition we may reduce to the case that $\beta^{*}$ and $\gamma^{*}$ are continuous. Take some $a \in E$ and some $x \in\left(\beta^{*}(a), \gamma^{*}(a)\right)$. Then we have $p\left(a^{\prime}, y\right)=p(a, y)(=f(\alpha, y))$ for all $a^{\prime} \in E$ sufficiently close to $a$, and all $y \in A$ sufficiently close to $x$. But 
$p\left(a^{\prime}, y\right)=p(a, y)$ gives $p\left(a^{\prime}-a, y\right)=0$, which can hold for at most $n$ values of $y$ in $A$ if $a^{\prime} \neq a$. This contradiction finishes the proof of the lemma.

While our main interest is in dense pairs of o-minimal expansions of real closed fields, the next lemma makes most of our work go through in the more general setting of this paper at no extra cost. The proof of this lemma uses the following special case of a theorem by Peterzil and Starchenko [6]:

Let $\mathcal{A}$ be an o-minimal expansion of an ordered vector space over an ordered field $F$, let $g: A^{p+1} \rightarrow A$ be definable in $\mathcal{A}$ and suppose that for infinitely many scalars $\lambda \in F$ there exists a tuple $a_{\lambda} \in A^{p}$ such that $g\left(a_{\lambda}, x\right)=\lambda x$ for infinitely many $x \in A$. Then there exists an interval $J$ in $\mathcal{A}$ and binary operations $\oplus, \otimes: J^{2} \rightarrow J$, definable in $\mathcal{A}$, that make $J$ into a real closed field whose ordering is the given ordering of $J$.

Lemma 1.2. Let $(\mathcal{B}, \mathcal{A})$ be a dense pair, let $f: B^{n+1} \rightarrow B$ be $A$-definable in $\mathcal{B}$, and let $b \in B \backslash A$. Then $f\left(A^{n} \times\{b\}\right) \neq B$.

Proof. We may as well assume that for each $k \in \mathbb{N}$ there is a tuple $a_{k} \in$ $A^{n}$ with $f\left(a_{k}, b\right)=k b$ since otherwise we would be done. Then $f\left(a_{k}, x\right)=k x$ for all $x \in I_{k}$, where $I_{k}$ is some interval in $\mathcal{B}$. Since $A$ is dense in $\mathcal{B}$ we may assume that $I_{k}$ is of the form $\left(c_{k}, d_{k}\right)$ with $c_{k}, d_{k} \in A$. Put $r_{k}:=\left(c_{k}+d_{k}\right) / 2$ and $s_{k}:=\left(d_{k}-c_{k}\right) / 2$, and note that $r_{k}, s_{k} \in A$. Let $g: A^{n+2} \rightarrow A$ be given by $g(u, v, x)=f(u, v+x)-f(u, v)$ for $u \in A^{n}$ and $v, x \in A$. Then $g$ is definable in $\mathcal{A}$ and $g\left(a_{k}, r_{k}, x\right)=k x$ for $-s_{k}<x<s_{k}$. By the result of Peterzil and Starchenko quoted above there exist then an interval $J$ in $\mathcal{A}$ and binary operations $\oplus, \otimes: J^{2} \rightarrow J$, definable in $\mathcal{A}$, that make $J$ into a real closed field whose ordering is the given ordering of $J$. Since $A$ is dense in $\mathcal{B}$ we may assume by translation that the extension $J_{\mathcal{B}}$ of this interval to $\mathcal{B}$ contains $b$. It now follows as in the proof of Lemma 1.1 (with the role of $A^{n+1}$ taken over by $J^{n+1}$ ) that there exist $a_{0}, \ldots, a_{n} \in J$ such that

$$
a_{0} \oplus\left(a_{1} \otimes b\right) \oplus \ldots \oplus\left(a_{n} \otimes b^{\otimes n}\right) \notin f\left(A^{n} \times\{b\}\right) .
$$

Corollary 1.3. Let $(\mathcal{B}, \mathcal{A})$ be a dense pair. Then each $\mathcal{A}$-small subset of $B$ is a proper subset of $B$.

Proof. This reduces to showing that if $g: B^{n} \rightarrow B$ is definable in $\mathcal{B}$, then $g\left(A^{n}\right) \neq B$. Given such a function $g$ there exists a function $f$ : $B^{n+p} \rightarrow B$ that is $A$-definable in $\mathcal{B}$ and a point $\left(b_{1}, \ldots, b_{p}\right) \in B^{p}$ such that $g(x)=f\left(x, b_{1}, \ldots, b_{p}\right)$ for all $x \in B^{n}$. By increasing $\mathcal{A}$ if necessary we may reduce to the case that $\mathcal{B}=\mathcal{A}\langle b\rangle$ for some $b \in B \backslash A$. Write each $b_{i}$ as $b_{i}=h_{i}(b)$ for some function $h_{i}: B \rightarrow B$ that is $A$-definable in $\mathcal{B}$. Replacing $f$ by the function

$$
(x, y) \mapsto f\left(x, h_{1}(y), \ldots, h_{p}(y)\right): B^{n+1} \rightarrow B
$$

and $\left(b_{1}, \ldots, b_{p}\right)$ by $b$ we reduce to the case treated in the previous lemma. 
REMARK 1.4. It is clear from the definitions that for any dense pair $(\mathcal{B}, \mathcal{A})$ the union of finitely many $\mathcal{A}$-small subsets of $B$ is again an $\mathcal{A}$-small subset of $B$.

LEMmA 1.5. If the dense pair $(\mathcal{B}, \mathcal{A})$ is $\kappa$-saturated where $\kappa$ is an infinite cardinal and $\kappa>|T|$, then $\operatorname{rk}(\mathcal{B} \mid \mathcal{A}) \geq \kappa$.

Proof. Let $(\mathcal{B}, \mathcal{A})$ be as in the hypothesis of the lemma. Let $E$ be a basis of $\mathcal{B}$ over $\mathcal{A}$, and suppose that $|E|<\kappa$. Let $\Gamma(v)$ be the set of all $L^{2}(E)$-formulas of the form

$$
\forall y_{1} \ldots \forall y_{n}\left(\left(U\left(y_{1}\right) \& \ldots \& U\left(y_{n}\right)\right) \rightarrow t\left(y_{1}, \ldots, y_{n}, e_{1}, \ldots, e_{p}\right) \neq v\right)
$$

where $t\left(y_{1}, \ldots, y_{n}, z_{1}, \ldots, z_{p}\right)$ is an $L$-term. By Corollary 1.3 and Remark 1.4 every finite subset of $\Gamma(v)$ is realized in $(\mathcal{B}, \mathcal{A})$. By saturation there exists $b \in B$ that realizes the type $\Gamma(v)$ in $(\mathcal{B}, \mathcal{A})$. Thus $b \notin \mathcal{A}\langle E\rangle$, contradicting $\mathcal{B}=\mathcal{A}\langle E\rangle$.

\section{Quantifier elimination}

2.1. Let $(\mathcal{B}, \mathcal{A}) \subseteq(\mathcal{D}, \mathcal{C}) \models T^{2}$, that is, the elementary pair $(\mathcal{B}, \mathcal{A})$ is a substructure of the elementary pair $(\mathcal{D}, \mathcal{C})$. Then $B \cap C=A$ and we have a diagram of (necessarily elementary) inclusions between models of $T$ :

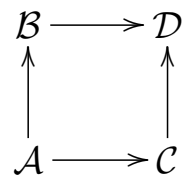

In this situation we say that $\mathcal{B}$ and $\mathcal{C}$ are free over $\mathcal{A}$ (in $\mathcal{D}$ ) if every set $Y \subseteq B$ that is independent over $\mathcal{A}$ is also independent over $\mathcal{C}$. Familiar results about pregeometries imply that then:

(i) $\mathcal{C}$ and $\mathcal{B}$ are free over $\mathcal{A}$ (symmetry);

(ii) if $Z \subseteq C$, then $(\mathcal{B}, \mathcal{A}) \subseteq(\mathcal{B}\langle Z\rangle, \mathcal{A}\langle Z\rangle) \subseteq(\mathcal{D}, \mathcal{C})$ and $\mathcal{B}\langle Z\rangle$ and $\mathcal{C}$ are free over $\mathcal{A}\langle Z\rangle$.

We mention here the following fact, although we do not use it further on.

FACT 2.2. Each elementary pair $(\mathcal{B}, \mathcal{A}) \models T^{2}$ can be embedded into a dense pair.

Proof. Let $a, b \in B$ with $a<b$. It suffices to show that $(\mathcal{B}, \mathcal{A})$ can be embedded into a model $\left(\mathcal{B}^{\prime}, \mathcal{A}^{\prime}\right)$ of $T^{2}$ such that $a<x<b$ for some $x \in A^{\prime}$. Take an elementary extension $\mathcal{B}\langle x\rangle$ generated over $\mathcal{B}$ by an element $x \notin B$ such that $a<x<b$. Then $\mathcal{B} \cap \mathcal{A}\langle x\rangle=\mathcal{A}$, hence $(\mathcal{B}\langle x\rangle, \mathcal{A}\langle x\rangle)$ is an extension of $(\mathcal{B}, \mathcal{A})$ with the desired property. 
Lemma 2.3. Let $(\mathcal{B}, \mathcal{A}) \preceq\left(\mathcal{B}^{*}, \mathcal{A}^{*}\right)$ be an elementary extension of models of $T^{2}$. Then in the diagram of (elementary) inclusions

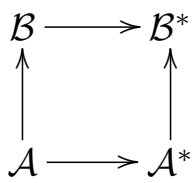

the structures $\mathcal{B}$ and $\mathcal{A}^{*}$ are free over $\mathcal{A}$.

Pro of. Let $b_{1}, \ldots, b_{n} \in B$ be independent over $\mathcal{A}$. We have to show that $b_{1}, \ldots, b_{n}$ remain independent over $\mathcal{A}^{*}$. If they did not, then after permuting $b_{1}, \ldots, b_{n}$ if necessary, we may assume that $b_{n}$ is dependent on $b_{1}, \ldots, b_{n-1}$ over $A^{*}$, so there are an $L$-formula $\phi\left(u_{1}, \ldots, u_{m}, v_{1}, \ldots, v_{n}\right)$, and an $m$-tuple $a^{*}$ from $\mathcal{A}^{*}$ such that

$$
\mathcal{B}^{*} \models \phi\left(a^{*}, b_{1}, \ldots, b_{n}\right) \& \exists ! v_{n} \phi\left(a^{*}, b_{1}, \ldots, b_{n-1}, v_{n}\right) .
$$

Since $(\mathcal{B}, \mathcal{A}) \preceq\left(\mathcal{B}^{*}, \mathcal{A}^{*}\right)$ it follows that for some $m$-tuple $a$ from $\mathcal{A}$ we have

$$
\mathcal{B}=\phi\left(a, b_{1}, \ldots, b_{n}\right) \& \exists ! v_{n} \phi\left(a, b_{1}, \ldots, b_{n-1}, v_{n}\right),
$$

which contradicts the assumption that $b_{1}, \ldots, b_{n}$ are independent over $\mathcal{A}$.

Lemma 2.4. If $(\mathcal{B}, \mathcal{A})$ is a dense pair, then $B \backslash A$ is dense in $\mathcal{B}$.

Proof. It is easy to reduce this to showing that, given any $\varepsilon>0$ in $A$ where $(\mathcal{B}, \mathcal{A})$ is a dense pair, there exists $\delta \in B \backslash A$ with $0<\delta<\varepsilon$. Take any $b \in B \backslash A$. Then $b-\varepsilon<a<b$ for some $a \in A$, hence $0<b-a<\varepsilon$, so $\delta:=b-a$ has the required property.

We can now prove completeness as well as Theorem 1 :

TheOREM 2.5. The theory $T^{\mathrm{d}}$ is complete. Each $L^{2}$-formula $\psi\left(y_{1}, \ldots\right.$, $\left.y_{n}\right)$ is equivalent in $T^{\mathrm{d}}$ to a boolean combination of formulas of the form

$$
\exists x_{1} \ldots \exists x_{m}\left(U\left(x_{1}\right) \& \ldots \& U\left(x_{m}\right) \& \phi\left(x_{1}, \ldots, x_{m}, y_{1}, \ldots, y_{n}\right)\right)
$$

where $\phi\left(x_{1}, \ldots, x_{m}, y_{1}, \ldots, y_{n}\right)$ is an L-formula.

Proof. Let $(\mathcal{B}, \mathcal{A})$ and $(\mathcal{D}, \mathcal{C})$ be $\kappa$-saturated models of $T^{\text {d }}$ where $\kappa>$ $|T|$. Let $\Gamma$ be the set of all isomorphisms $i:\left(\mathcal{B}^{\prime}, \mathcal{A}^{\prime}\right) \cong\left(\mathcal{D}^{\prime}, \mathcal{C}^{\prime}\right)$ between substructures $\left(\mathcal{B}^{\prime}, \mathcal{A}^{\prime}\right)$ of $(\mathcal{B}, \mathcal{A})$ and $\left(\mathcal{D}^{\prime}, \mathcal{C}^{\prime}\right)$ of $(\mathcal{D}, \mathcal{C})$ such that $\left|B^{\prime}\right|<\kappa$, $\left|D^{\prime}\right|<\kappa, \mathcal{B}^{\prime}$ and $\mathcal{A}$ are free over $\mathcal{A}^{\prime}$, and $\mathcal{D}^{\prime}$ and $\mathcal{C}$ are free over $\mathcal{C}^{\prime}$. The completeness of $T^{\mathrm{d}}$ will follow if we can show that $(\mathcal{B}, \mathcal{A})$ and $(\mathcal{D}, \mathcal{C})$ are elementarily equivalent, and this in turn follows from

ClaIm. The collection $\Gamma$ has the back-and-forth property.

Pro of. Let $i:\left(\mathcal{B}^{\prime}, \mathcal{A}^{\prime}\right) \cong\left(\mathcal{D}^{\prime}, \mathcal{C}^{\prime}\right)$ belong to $\Gamma$. Consider first an element $a \in A \backslash A^{\prime}$. Then we take an element $c \in C \backslash C^{\prime}$ such that the cuts realized by $a$ in $\mathcal{B}^{\prime}$ and by $c$ in $\mathcal{D}^{\prime}$ correspond via $i$. Then $i$ extends to an isomorphism 
$j:\left(\mathcal{B}^{\prime}\langle a\rangle, \mathcal{A}^{\prime}\langle a\rangle\right) \cong\left(\mathcal{D}^{\prime}\langle c\rangle, \mathcal{C}^{\prime}\langle c\rangle\right)$ with $j(a)=c$, and clearly $j \in \Gamma$. Now consider more generally an element $b \in B \backslash B^{\prime}$.

CAse 1: $b \in \mathcal{B}^{\prime}\langle A\rangle$. Then take elements $a_{1}, \ldots, a_{k} \in A$ such that $b \in$ $\mathcal{B}^{\prime}\left\langle a_{1}, \ldots, a_{k}\right\rangle$. Applying the above construction several times we obtain elements $c_{1}, \ldots, c_{k} \in C$ such that $i$ extends to an isomorphism

$$
\left(\mathcal{B}^{\prime}\left\langle a_{1}, \ldots, a_{k}\right\rangle, \mathcal{A}^{\prime}\left\langle a_{1}, \ldots, a_{k}\right\rangle\right) \cong\left(\mathcal{D}^{\prime}\left\langle c_{1}, \ldots, c_{k}\right\rangle, \mathcal{C}^{\prime}\left\langle c_{1}, \ldots, c_{k}\right\rangle\right)
$$

in $\Gamma$, which includes $b$ in its domain.

CASE 2: $b \notin \mathcal{B}^{\prime}\langle A\rangle$. Then $\left(\mathcal{B}^{\prime}\langle b\rangle, \mathcal{A}^{\prime}\right) \subseteq(\mathcal{B}, \mathcal{A})$, and $\mathcal{B}^{\prime}\langle b\rangle$ and $\mathcal{A}$ are free over $\mathcal{A}^{\prime}$, as is easily checked. By Lemma 1.5 we have $\mathcal{D}^{\prime}\langle C\rangle=\mathcal{C}\left\langle D^{\prime}\right\rangle \neq \mathcal{D}$, and thus by Lemma 2.4 and saturation there exists an element $d \in \mathcal{D} \backslash \mathcal{D}^{\prime}\langle C\rangle$ such that the cuts realized by $b$ in $\mathcal{B}^{\prime}$ and by $d$ in $\mathcal{D}^{\prime}$ correspond via $i$. Thus $i$ extends to an isomorphism $j:\left(\mathcal{B}^{\prime}\langle b\rangle, \mathcal{A}^{\prime}\right) \cong\left(\mathcal{D}^{\prime}\langle d\rangle, \mathcal{C}^{\prime}\right)$ with $j(b)=d$, and clearly $j$ belongs to $\Gamma$.

This proof of the completeness of $T^{\mathrm{d}}$ provides the extra information that if $i \in \Gamma$ and $\left(b_{1}, \ldots, b_{N}\right)$ is a tuple from the domain of $i$, then $\left(b_{1}, \ldots, b_{N}\right)$ realizes the same type in $(\mathcal{B}, \mathcal{A})$ as $\left(i\left(b_{1}\right), \ldots, i\left(b_{N}\right)\right)$ in $(\mathcal{D}, \mathcal{C})$. We shall use this fact below in the proof of the second part of the theorem where we fix the tuple of free variables $y=\left(y_{1}, \ldots, y_{n}\right)$. Call an $L^{2}$-formula $\psi(y)$ special if it is of the form $\exists x(U(x) \& \phi(x, y))$ as described in the theorem, where $x=\left(x_{1}, \ldots, x_{m}\right)$ and $U(x)$ abbreviates $U\left(x_{1}\right) \& \ldots \& U\left(x_{m}\right)$. Let $b=\left(b_{1}, \ldots, b_{n}\right) \in B^{n}$ and $d=\left(d_{1}, \ldots, d_{n}\right) \in D^{n}$ satisfy the same special formulas in $(\mathcal{B}, \mathcal{A})$ and $(\mathcal{D}, \mathcal{C})$ respectively. It suffices to show that then $b$ and $d$ realize the same types in $(\mathcal{B}, \mathcal{A})$ and $(\mathcal{D}, \mathcal{C})$ respectively. Let $\operatorname{rk}(\mathcal{A}\langle b\rangle \mid \mathcal{A})=$ $r$. By permuting coordinates we may assume that $b_{1}, \ldots, b_{r}$ are independent over $\mathcal{A}$. Then also $\operatorname{rk}(\mathcal{C}\langle d\rangle \mid C)=r$ and $d_{1}, \ldots, d_{r}$ are independent over $\mathcal{C}$. (For example, suppose $d_{1}, \ldots, d_{r}$ were dependent over $\mathcal{C}$. Then $r>0$ and, say, $d_{r}=f\left(c, d_{1}, \ldots, d_{r-1}\right)$ for some $c \in C^{m}$ and some function $f: D^{m+n} \rightarrow D$ which is 0-definable in $\mathcal{D}$. Then the hypothesis on $b$ and $d$ implies that also $b_{r}=f\left(a, b_{1}, \ldots, b_{r-1}\right)$ for some $a \in A^{m}$, where, abusing notation, $f$ now denotes the corresponding function $B^{m+n} \rightarrow B$ defined in $\mathcal{B}$. But this contradicts the independence of $b_{1}, \ldots, b_{r}$ over $\mathcal{A}$.)

Take a tuple $a=\left(a_{1}, \ldots, a_{m}\right) \in A^{m}$ such that $\operatorname{rk}\left(\mathcal{A}^{\prime}\langle b\rangle, \mathcal{A}^{\prime}\right)=r$ where $\mathcal{A}^{\prime}$ is the definable closure of $\left\{a_{1}, \ldots, a_{m}\right\}$ in $\mathcal{A}$. Note that then $\left(\mathcal{A}^{\prime}\langle b\rangle, \mathcal{A}^{\prime}\right) \subseteq$ $(\mathcal{B}, \mathcal{A})$ and that $\mathcal{A}^{\prime}\langle b\rangle$ and $\mathcal{A}$ are free over $\mathcal{A}^{\prime}$. If $\phi_{1}(x, y), \ldots, \phi_{k}(x, y)$ are $L$-formulas such that $\mathcal{B}=\phi_{j}(a, b)$ for $j=1, \ldots, k$, then by the hypothesis on $b$ and $d$ we have $(\mathcal{D}, \mathcal{C}) \models \bigwedge_{j=1}^{k}\left(U(x) \& \phi_{i}(x, d)\right)$. Hence by saturation there is a tuple $c=\left(c_{1}, \ldots, c_{m}\right) \in C^{m}$ such that $(a, b)$ and $(c, d)$ realize the same types in $\mathcal{B}$ and $\mathcal{D}$ respectively. One easily checks that then $\operatorname{rk}\left(\mathcal{C}^{\prime}\langle d\rangle, \mathcal{C}^{\prime}\right)=r$ where $\mathcal{C}^{\prime}$ is the definable closure of $\left\{c_{1}, \ldots, c_{m}\right\}$ in $\mathcal{C}$. Hence $\left(\mathcal{C}^{\prime}\langle b\rangle, \mathcal{C}^{\prime}\right) \subseteq$ $(\mathcal{D}, \mathcal{C})$ and $\mathcal{C}^{\prime}\langle d\rangle$ and $\mathcal{C}$ are free over $\mathcal{C}^{\prime}$. Thus we obtain an isomorphism 
$i:\left(\mathcal{A}^{\prime}\langle b\rangle, \mathcal{A}^{\prime}\right) \cong\left(\mathcal{C}^{\prime}\langle b\rangle, \mathcal{C}^{\prime}\right)$ in $\Gamma$ with $i(a, b)=(c, d)$. Therefore $b$ and $d$ realize the same types in $(\mathcal{B}, \mathcal{A})$ and $(\mathcal{D}, \mathcal{A})$ respectively.

Corollary 2.6. Let $(\mathcal{B}, \mathcal{A})$ be a dense pair and suppose $Y \subseteq B^{n}$ is $A_{0}$-definable in $(\mathcal{B}, \mathcal{A})$ where $A_{0} \subseteq A$. Then $Y \cap A^{n}$ is $A_{0}$-definable in $\mathcal{A}$.

Proof. By the theorem this reduces to the case of $Y$ defined in $(\mathcal{B}, \mathcal{A})$ by a formula $\exists x(U(x) \& \phi(x, y))$ where $\phi(x, y)$ is an $L\left(A_{0}\right)$-formula. Then $Y \cap A^{n}$ is defined in $\mathcal{A}$ by the $L\left(A_{0}\right)$-formula $\exists x \phi(x, y)$.

The next corollaries record some consequences of the proof of Theorem 2.5 .

Corollary 2.7. Let $(\mathcal{B}, \mathcal{A})$ and $\left(\mathcal{B}^{\prime}, \mathcal{A}^{\prime}\right)$ be dense pairs such that $\left(\mathcal{B}^{\prime}, \mathcal{A}^{\prime}\right)$ $\subseteq(\mathcal{B}, \mathcal{A})$ and $\mathcal{B}^{\prime}$ and $\mathcal{A}$ are free over $\mathcal{A}^{\prime}$ in $\mathcal{B}$. Then $\left(\mathcal{B}^{\prime}, \mathcal{A}^{\prime}\right) \preceq(\mathcal{B}, \mathcal{A})$.

Proof. Take some cardinal $\kappa>\max \left(\left|B^{\prime}\right|,|T|\right)$ and some $\kappa$-saturated elementary extension $(\mathcal{D}, \mathcal{C})$ of $\left(\mathcal{B}^{\prime}, \mathcal{A}^{\prime}\right)$. Then $\mathcal{B}^{\prime}$ and $\mathcal{C}$ are free over $\mathcal{A}^{\prime}$ by Lemma 2.3. By that same lemma we may assume, by passing to an elementary extension of $(\mathcal{B}, \mathcal{A})$ if necessary, that $(\mathcal{B}, \mathcal{A})$ is $\kappa$-saturated. By the proof of 2.5 any tuple in $\left(\mathcal{B}^{\prime}, \mathcal{A}^{\prime}\right)$ realizes the same type in $(\mathcal{B}, \mathcal{A})$ as in $(\mathcal{D}, \mathcal{C})$. Since $\left(\mathcal{B}^{\prime}, \mathcal{A}^{\prime}\right) \preceq(\mathcal{D}, \mathcal{C})$ this gives $\left(\mathcal{B}^{\prime}, \mathcal{A}^{\prime}\right) \preceq(\mathcal{B}, \mathcal{A})$.

Corollary 2.8. Let $(\mathcal{B}, \mathcal{A})=T^{2}$ have extensions $\left(\mathcal{B}_{1}, \mathcal{A}_{1}\right)=T^{\mathrm{d}}$ and $\left(\mathcal{B}_{2}, \mathcal{A}_{2}\right) \models T^{\mathrm{d}}$ such that $\mathcal{B}$ and $\mathcal{A}_{1}$ are free over $\mathcal{A}$, and $\mathcal{B}$ and $\mathcal{A}_{2}$ are free over $\mathcal{A}$ in $\mathcal{B}_{2}$. See the inclusion diagram:

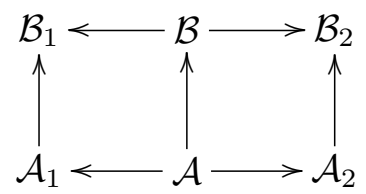

Then $\left(\mathcal{B}_{1}, \mathcal{A}_{1}\right) \equiv_{B}\left(\mathcal{B}_{2}, \mathcal{A}_{2}\right)$, that is, $\left(\mathcal{B}_{1}, \mathcal{A}_{1}\right)$ and $\left(\mathcal{B}_{2}, \mathcal{A}_{2}\right)$ satisfy the same sentences with parameters from $B$. More generally, if $a_{1} \in\left(A_{1}\right)^{n}$ and $a_{2} \in$ $\left(A_{2}\right)^{n}$ realize the same types over $B$ in $\mathcal{B}_{1}$ and $\mathcal{B}_{2}$ respectively, then they even realize the same types over $B$ in $\left(\mathcal{B}_{1}, \mathcal{A}_{1}\right)$ and $\left(\mathcal{B}_{2}, \mathcal{A}_{2}\right)$ respectively.

Proof. By passing to suitable elementary extensions we may assume that $\left(\mathcal{B}_{k}, \mathcal{A}_{k}\right)$ is $\kappa$-saturated with $\kappa>\max (|B|,|T|), k=1,2$. Note that $\mathcal{B}\left\langle a_{k}\right\rangle$ and $\mathcal{A}_{k}$ are free over $\mathcal{A}\left\langle a_{k}\right\rangle(k=1,2)$. The hypothesis on $a_{1}$ and $a_{2}$ implies that there exists an isomorphism $i:\left(\mathcal{B}\left\langle a_{1}\right\rangle, \mathcal{A}\left\langle a_{1}\right\rangle\right) \cong\left(\mathcal{B}\left\langle a_{2}\right\rangle, \mathcal{A}\left\langle a_{2}\right\rangle\right)$ that is the identity on $B$ with $i\left(a_{1}\right)=a_{2}$. Then Theorem 2.5 and its proof tell us that $a_{1}$ and $a_{2}$ realize the same types over $B$ in $\left(\mathcal{B}_{1}, \mathcal{A}_{1}\right)$ and $\left(\mathcal{B}_{2}, \mathcal{A}_{2}\right)$ respectively.

Corollary 2.9. Let $\left(\mathcal{B}_{1}, \mathcal{A}_{1}\right)$ and $\left(\mathcal{B}_{2}, \mathcal{A}_{2}\right)$ be dense pairs and let $\mathcal{A}_{1}$ and $\mathcal{A}_{2}$ have a common (elementary) substructure $\mathcal{A}$. Suppose $b_{1} \in B_{1} \backslash A_{1}$ and $b_{2} \in B_{2} \backslash A_{2}$ realize the same cut in $\mathcal{A}$. Then they realize the same types over $A$ in $\left(\mathcal{B}_{1}, \mathcal{A}_{1}\right)$ and $\left(\mathcal{B}_{2}, \mathcal{A}_{2}\right)$ respectively. 
Proof. By passing to a suitable elementary extension we may assume that $\left(\mathcal{B}_{k}, \mathcal{A}_{k}\right)$ is $\kappa$-saturated with $\kappa>\max (|A|,|T|), k=1,2$. Consider the diagram

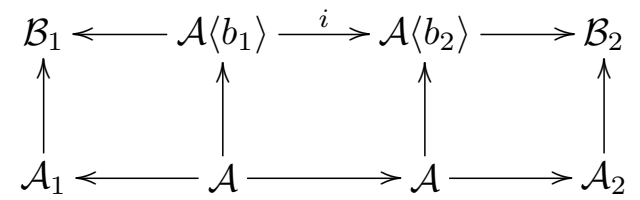

where $i: \mathcal{A}\left\langle b_{1}\right\rangle \rightarrow \mathcal{A}\left\langle b_{2}\right\rangle$ is the isomorphism over $\mathcal{A}$ that sends $b_{1}$ to $b_{2}$. Except for $i$ the arrows in this diagram are inclusions. In the leftmost rectangle, $\mathcal{A}\left\langle b_{1}\right\rangle$ and $\mathcal{A}_{1}$ are free over $\mathcal{A}$, while in the rightmost rectangle, $\mathcal{A}\left\langle b_{2}\right\rangle$ and $\mathcal{A}_{2}$ are free over $\mathcal{A}$. Hence by Theorem 2.5 and its proof $b_{1}$ and $b_{2}$ realize the same types over $A$ in $\left(\mathcal{B}_{1}, \mathcal{A}_{1}\right)$ and $\left(\mathcal{B}_{2}, \mathcal{A}_{2}\right)$ respectively.

For Theorem 2 we also need the following:

Lemma 2.10. Let $Z \subseteq B^{n}$ be definable in $\mathcal{B}$. Then $Z$ is a finite union of sets of the form $\left\{z \in B^{n}: f(b, z)=0, g(b, z)>0\right\}$ where $b \in B^{m}$ and $f, g: B^{m+n} \rightarrow B$ are continuous and 0 -definable in $\mathcal{B}$.

Proof. Write $Z=Z_{b}^{\prime}$ where $b \in B^{m}$ and $Z^{\prime} \subseteq B^{m+n}$ is 0-definable in $\mathcal{B}$. By decomposing $Z^{\prime}$ into 0-definable cells we may reduce to the case of $Z^{\prime}$ being a 0-definable cell (with respect to $\mathcal{B}$ ). Then the functions $f, g$ : $B^{m+n} \rightarrow B$ given by $f(x)=d\left(x, \operatorname{cl}\left(Z^{\prime}\right)\right)$ and $g(x)=d\left(x, \operatorname{cl}\left(Z^{\prime}\right) \backslash Z^{\prime}\right)$ are continuous and 0 -definable in $\mathcal{B}$. (By convention we let $d(x, \emptyset):=1$.) As a cell $Z^{\prime}$ is locally closed, and thus $\mathrm{cl}\left(Z^{\prime}\right) \backslash Z^{\prime}$ is closed. Hence $Z^{\prime}=\{x \in$ $\left.B^{m+n}: f(x)=0, g(x)>0\right\}$. Thus $Z=\left\{z \in B^{n}: f(b, z)=0, g(b, z)>0\right\}$.

2.11. Proof of Theorem 2. We are given $(\mathcal{B}, \mathcal{A}) \models T^{\mathrm{d}}$ and a set $Y \subseteq A^{n}$. $(1) \Rightarrow(2)$. We assume here that $Y$ is definable in $(\mathcal{B}, \mathcal{A})$ and have to show that $Y=Z \cap A^{n}$ for some set $Z \subseteq B^{n}$ that is definable in $\mathcal{B}$. Let $\phi(y)$ be an $L^{2}(B)$-formula defining $Y$ in $(\mathcal{B}, \mathcal{A}), y=\left(y_{1}, \ldots, y_{n}\right)$. We have to show there is an $L(B)$-formula $\psi(y)$ such that $(\mathcal{B}, \mathcal{A}) \models U(y) \rightarrow(\phi(y) \leftrightarrow \psi(y))$. By a standard model-theoretic argument this will be the case if for any two elementary extensions $\left(\mathcal{B}_{1}, \mathcal{A}_{1}\right)$ and $\left(\mathcal{B}_{2}, \mathcal{A}_{2}\right)$ of $(\mathcal{B}, \mathcal{A})$ and any two $n$-tuples $a_{1} \in\left(A_{1}\right)^{n}$ and $a_{2} \in\left(A_{2}\right)^{n}$ that realize the same types over $B$ (in their ambient models $\mathcal{B}_{1}$ and $\mathcal{B}_{2}$ respectively) we have

$$
\left(\mathcal{B}_{1}, \mathcal{A}_{1}\right) \models \phi\left(a_{1}\right) \Leftrightarrow\left(\mathcal{B}_{2}, \mathcal{A}_{2}\right) \models \phi\left(a_{2}\right) .
$$

But this is immediate from 2.8 .

$(2) \Rightarrow(3)$. We assume $Y=Z \cap A^{n}$ with $Z \subseteq B^{n}$ definable in $\mathcal{B}$. We have to show that $Y$ is definable in $\left(\mathcal{A},(A \cap(0, b))_{0<b \in B}\right)$. By Lemma 2.10 we may reduce to the case of either $Z=\left\{z \in B^{n}: f(b, z)=0\right\}$ or $Z=\{z \in$ $\left.B^{n}: f(b, z)>0\right\}$ where $f: B^{m+n} \rightarrow B$ is continuous and 0-definable in $\mathcal{B}$, and $b=\left(b_{1}, \ldots, b_{m}\right) \in B^{m}$. Assume $Z=\left\{z \in B^{n}: f(b, z)=0\right\}$. (The other 
case is handled similarly.) Then by continuity of $f$ and density of $A$ in $\mathcal{B}$ we have the following equivalence for elements $y \in A^{m}$ :

$$
\begin{aligned}
y \in Y \Leftrightarrow & \text { for each } \varepsilon>0 \text { in } A \text { there is } a=\left(a_{1}, \ldots, a_{m}\right) \in A^{m} \text { such that } \\
& a_{1}<b_{1}, \ldots, a_{m}<b_{m} \text { and for all } a^{\prime}=\left(a_{1}^{\prime}, \ldots, a_{m}^{\prime}\right) \in A^{m}, \\
& \text { if } a_{1}<a_{1}^{\prime}<b_{1}, \ldots, a_{m}<a_{m}^{\prime}<b_{m} \text {, then }\left|f\left(a^{\prime}, y\right)\right|<\varepsilon .
\end{aligned}
$$

Clearly, the set of all $y \in A^{m}$ that satisfy the right-hand condition is definable in the structure $\left(\mathcal{A},\left(A \cap(0, b)_{0<b \in B}\right)\right.$, and thus $Y$ is definable in this structure.

$$
(3) \Rightarrow(1) \text { is obvious. }
$$

3. Definable closure and definable functions. In this section we prove Theorem 3, for which we need results on "definable closure".

Lemma 3.1. Let $(\mathcal{B}, \mathcal{A}) \models T^{\mathrm{d}}$. Then the set $A$ is definably closed in $(\mathcal{B}, \mathcal{A})$.

Proof. Let $b \in B \backslash A$. We will show that $b$ is not $A$-definable in $(\mathcal{B}, \mathcal{A})$. Take some $\kappa$-saturated elementary extension $\left(\mathcal{B}^{*}, \mathcal{A}^{*}\right)$ of $(\mathcal{B}, \mathcal{A})$ with $\kappa>$ $\max (|B|,|T|)$. Then there exists $b^{*} \in B^{*} \backslash A^{*}$ such that $b^{*} \neq b$, and $b^{*}$ and $b$ realize the same cut in $\mathcal{A}$. Corollary 2.9 implies that then $b^{*}$ and $b$ realize the same type over $A$ in $\left(\mathcal{B}^{*}, \mathcal{A}^{*}\right)$. Thus $b$ is not $A$-definable in $\left(\mathcal{B}^{*}, \mathcal{A}^{*}\right)$, and therefore not $A$-definable in $(\mathcal{B}, \mathcal{A})$.

Lemma 3.2. Let $(\mathcal{B}, \mathcal{A}) \models T^{\mathrm{d}}$, and $\mathcal{A}_{0} \preceq \mathcal{A}$. Then $A_{0}$ is definably closed in $(\mathcal{B}, \mathcal{A})$.

Proof. Let $a \in B$ be in the definable closure of $A_{0}$ in $(\mathcal{B}, \mathcal{A})$. By the previous lemma we have $a \in A$. Then 2.6 implies that $a \in A_{0}$.

Proposition 3.3. Let $(\mathcal{B}, \mathcal{A}) \subseteq\left(\mathcal{B}^{*}, \mathcal{A}^{*}\right) \models T^{\mathrm{d}}$ and suppose that $\mathcal{B}$ and $\mathcal{A}^{*}$ are free over $\mathcal{A}$. Then the set $B$ is definably closed in $\left(\mathcal{B}^{*}, \mathcal{A}^{*}\right)$.

Proof. The previous lemma shows this is true if $A=B$. Suppose that $A \neq B$. Then $\left(\mathcal{A}^{*}\langle B\rangle, \mathcal{A}^{*}\right) \preceq\left(\mathcal{B}^{*}, \mathcal{A}^{*}\right)$, so the definable closure of $B$ in $\left(\mathcal{B}^{*}, \mathcal{A}^{*}\right)$ is contained in (the underlying set of) $\mathcal{A}^{*}\langle B\rangle=\mathcal{B}\left\langle A^{*}\right\rangle$. Let $b^{*}$ be an element of this definable closure. Take $k \in \mathbb{N}$ minimal such that there exists $a^{*}=\left(a_{1}^{*}, \ldots, a_{k}^{*}\right) \in\left(A^{*}\right)^{k}$ with $b^{*} \in \mathcal{B}\left\langle a^{*}\right\rangle$, and fix such a tuple $a^{*}$. It suffices to show that $k=0$. Suppose $k>0$. The minimality of $k$ implies that $a_{1}^{*}, \ldots, a_{k}^{*}$ are independent over $\mathcal{B}$. Let $f:\left(B^{*}\right)^{k} \rightarrow B^{*}$ be $B$-definable in $\mathcal{B}^{*}$ such that $f\left(a^{*}\right)=b^{*}$. Take a finite partition $\Pi$ of $\left(B^{*}\right)^{k}$ into $B$-definable cells such that if $E \in \Pi$ is open, then either $f \mid E$ is strictly increasing in its $k$ th variable, or $f \mid E$ is independent of its $k$ th variable, or $f \mid E$ is strictly decreasing in its $k$ th variable. (The existence of such a partition is a routine exercise in cell decomposition which we leave to the reader.) Note that $a^{*}$ necessarily belongs to an open cell $E \in \Pi$. Passing to a suitable elementary 
extension if necessary we may assume that $\left(\mathcal{B}^{*}, \mathcal{A}^{*}\right)$ is $\kappa$-saturated with $\kappa>\max (|B|,|T|)$.

CASE 1: The function $f \mid E$ does not depend on the last variable. Then $b^{*}=f\left(a^{*}\right)=g\left(a_{1}^{*}, \ldots, a_{k-1}^{*}\right)$ for some function $g:\left(B^{*}\right)^{k-1} \rightarrow B^{*}$ that is $B$-definable in $\mathcal{B}^{*}$, contradicting the minimality of $k$.

CASE 2: The function $f \mid E$ is strictly increasing in the last variable. Put $\mathcal{A}^{\prime}:=\mathcal{A}\left\langle a_{1}^{*}, \ldots, a_{k-1}^{*}\right\rangle$ and $\mathcal{B}^{\prime}:=\mathcal{B}\left\langle a_{1}^{*}, \ldots, a_{k-1}^{*}\right\rangle$. By saturation we can take an element $a_{k} \neq a_{k}^{*}$ in $A^{*}$ that realizes the same cut in $\mathcal{B}^{\prime}$ as $a_{k}^{*}$. Corollary 2.8 implies that then $a_{k}$ and $a_{k}^{*}$ realize the same type over $B^{\prime}$ in $\left(\mathcal{B}^{*}, \mathcal{A}^{*}\right)$, in particular $\left(a_{1}^{*}, \ldots, a_{k-1}^{*}, a_{k}\right) \in E$ and $b^{*}=f\left(a_{1}^{*}, \ldots, a_{k-1}^{*}, a_{k}\right)$ (using the fact that $b^{*}$ is $B$-definable in $\left.\left(\mathcal{B}^{*}, \mathcal{A}^{*}\right)\right)$. This last equality contradicts the hypothesis of Case 2 .

CASE 3: The function $f \mid E$ is strictly decreasing in the last variable. We proceed as in Case 2.

We can now prove Theorem 3, which we reformulate here as three separate corollaries.

Corollary 3.4. Let the function $F: B \rightarrow B$ be definable in the dense pair $(\mathcal{B}, \mathcal{A})$. Then $F$ agrees off some $\mathcal{A}$-small subset of $B$ with a function $\widehat{F}: B \rightarrow B$ that is definable in $\mathcal{B}$.

Proof. Let $\left(\mathcal{B}^{*}, \mathcal{A}^{*}\right)$ be a $\kappa$-saturated elementary extension of $(\mathcal{B}, \mathcal{A})$ with $\kappa>\max (|B|,|T|)$. Let $b^{*} \in B^{*}$ and note that $b^{*}$ lies outside $X^{*}$ for all $\mathcal{A}$-small sets $X \subseteq B$ if and only if $b^{*} \notin \mathcal{B}\left\langle A^{*}\right\rangle$. Here $X^{*}$ denotes the subset of $B^{*}$ defined in $\left(\mathcal{B}^{*}, \mathcal{A}^{*}\right)$ by any $L^{2}(B)$-formula that defines $X$ in $(\mathcal{B}, \mathcal{A})$. By familiar model-theoretic arguments it suffices to show that if $b^{*} \in \mathcal{B}^{*} \backslash \mathcal{B}\left\langle A^{*}\right\rangle$, then $F\left(b^{*}\right) \in \mathcal{B}\left\langle b^{*}\right\rangle$. But for such $b^{*}$ the models $\mathcal{B}\left\langle b^{*}\right\rangle$ and $\mathcal{A}^{*}$ are free over $\mathcal{A}$ in $\mathcal{B}^{*}$. Thus by the last proposition the underlying set of $\mathcal{B}\left\langle b^{*}\right\rangle$ is definably closed in $\left(\mathcal{B}^{*}, \mathcal{A}^{*}\right)$, which implies $F\left(b^{*}\right) \in \mathcal{B}\left\langle b^{*}\right\rangle$, as desired.

Applying this result to the characteristic function of a set $S \subseteq B$ we obtain

Corollary 3.5. If $S \subseteq B$ is definable in the dense pair $(\mathcal{B}, \mathcal{A})$, then $S \backslash X=S^{\prime} \backslash X$ for some $\mathcal{A}$-small set $X \subseteq B$ and some $S^{\prime} \subseteq B$ that is definable in $\mathcal{B}$.

Corollary 3.6. Let $(\mathcal{B}, \mathcal{A})=T^{\mathrm{d}}$, and let $f: A^{n} \rightarrow A$ be definable in $(\mathcal{B}, \mathcal{A})$. Then $f$ is given piecewise by functions definable in $\mathcal{A}$ : there are functions $f_{1}, \ldots, f_{k}: A^{n} \rightarrow A$ definable in $\mathcal{A}$ such that for each $a \in A^{n}$ we have $f(a)=f_{i}(a)$ for some $i \in\{1, \ldots, k\}$.

Proof. By a familiar compactness argument it suffices to show that, given an elementary extension $\left(\mathcal{B}^{*}, \mathcal{A}^{*}\right)$ of $(\mathcal{B}, \mathcal{A})$ and a point $a^{*} \in\left(A^{*}\right)^{n}$, 
we have $f\left(a^{*}\right) \in \mathcal{A}\left\langle a^{*}\right\rangle$. It follows from 2.1 and Lemma 2.3 that $\mathcal{B}\left\langle a^{*}\right\rangle$ and $\mathcal{A}^{*}$ are free over $\mathcal{A}\left\langle a^{*}\right\rangle$. Hence by Proposition 3.3 the underlying set of $\mathcal{B}\left\langle a^{*}\right\rangle$ is definably closed in $\left(\mathcal{B}^{*}, \mathcal{A}^{*}\right)$. Thus $f\left(a^{*}\right) \in \mathcal{B}\left\langle a^{*}\right\rangle$. Since also $f\left(a^{*}\right) \in A^{*}$ and $\mathcal{B}\left\langle a^{*}\right\rangle \cap \mathcal{A}^{*}=\mathcal{A}\left\langle a^{*}\right\rangle$ we may conclude that $f\left(a^{*}\right) \in \mathcal{A}\left\langle a^{*}\right\rangle$.

4. Definable sets in one variable, and open definable sets. In this section we fix a dense pair $(\mathcal{B}, \mathcal{A})$. We will prove Theorems 4 and 5 after some preparations.

Lemma 4.1. No interval in $\mathcal{B}$ is $\mathcal{A}$-small.

Proof. Let $I$ be an interval in $\mathcal{B}$, and $f: B^{m} \rightarrow B$ definable in $\mathcal{B}$. We have to show that $I \nsubseteq f\left(A^{m}\right)$. Let $\left(\mathcal{B}^{*}, \mathcal{A}^{*}\right)$ be a $\kappa$-saturated elementary extension of $(\mathcal{B}, \mathcal{A})$ with $\kappa>\max (|B|,|T|)$, and let $I^{*}$ and $f^{*}$ be the obvious extensions of $I$ and $f$ to $\left(\mathcal{B}^{*}, \mathcal{A}^{*}\right)$. Then $f^{*}\left(\left(A^{*}\right)^{m}\right) \subseteq \mathcal{A}^{*}\langle B\rangle$. By Lemmas 1.5 and 2.4 there is an element in $I^{*}$ that does not lie in $\mathcal{A}^{*}\langle B\rangle$, and thus not in $f^{*}\left(\left(A^{*}\right)^{m}\right)$. Hence $I$ contains an element that lies outside $f\left(A^{m}\right)$.

Lemma 4.2. Let $S \subseteq B^{m}$ be definable in $\mathcal{B}$, and let the map $g=$ $\left(g_{1}, \ldots, g_{k}\right): B^{m} \rightarrow B^{k}$ be definable in $\mathcal{B}$. Then there is a set $S^{\prime} \subseteq S$, definable in $\mathcal{B}$, such that

$$
A^{m} \cap S \cap g^{-1}\left(A^{k}\right)=A^{m} \cap S^{\prime} .
$$

REMARK. This lemma goes through (with the same proof) for $(\mathcal{B}, \mathcal{A}) \models$ $T^{2}$, and does not need the stronger assumption $(\mathcal{B}, \mathcal{A}) \models T^{\mathrm{d}}$.

Pro of (of Lemma 4.2). The case of $S=\emptyset$ is trivial, so we assume from now on that $S \neq \emptyset$, and we proceed by induction on the triple $(m, d, k) \in \mathbb{N}^{3}$ where $d:=\operatorname{dim}(S)$ and $\mathbb{N}^{3}$ is ordered lexicographically. The desired result is obvious if $m=0$, or $d=0$ (that is, $S$ is finite), or $k=0$. So we assume that $m>0, d>0$ and $k>0$, and that the desired result holds for lower values of ( $m, d, k)$ (inductive hypothesis). The case $k>1$ reduces to the case $k=1$ (with same $m$ and $d$ ) by means of

$$
A^{m} \cap S \cap g^{-1}\left(A^{k}\right)=\left(A^{m} \cap S \cap g_{1}^{-1}\left(A^{k}\right)\right) \cap \ldots \cap\left(A^{m} \cap S \cap g_{k}^{-1}\left(A^{k}\right)\right) .
$$

Thus we assume below that $k=1$.

Suppose that $d<m$. Decomposing $S$ into cells we may then reduce to the case of $S$ being a cell; thus there are $i_{1}, \ldots, i_{d}$ with $1 \leq i_{1}<\ldots<i_{d} \leq m$ such that the projection map $\lambda:\left(x_{1}, \ldots, x_{m}\right) \mapsto\left(x_{i_{1}}, \ldots, x_{i_{d}}\right): B^{m} \rightarrow B^{d}$ maps $S$ homeomorphically onto an open cell $\lambda(S)$ in $B^{d}$. Take a map $\mu$ : $B^{d} \rightarrow B^{m}$ that is definable in $\mathcal{B}$ and inverse to $\lambda \mid S$, that is, $\mu(\lambda(x))=x$ for $x \in S$. By the inductive hypothesis there is a set $S^{\prime \prime} \subseteq \lambda(S)$, definable in $\mathcal{B}$, such that

$$
A^{d} \cap \lambda(S) \cap \mu^{-1}\left(A^{m}\right) \cap(g \circ \mu)^{-1}(A)=A^{d} \cap S^{\prime \prime} .
$$


Then $\mu\left(S^{\prime \prime}\right) \subseteq S$ and $A^{m} \cap S \cap g^{-1}(A)=A^{m} \cap \mu\left(S^{\prime \prime}\right)$, and we are done. Thus for the rest of the proof we assume $d=m$.

Take a function $G: B^{m+n} \rightarrow B$ that is $A$-definable in $\mathcal{B}$, and a point $b=\left(b_{1}, \ldots, b_{n}\right) \in B^{n}$ such that $g(x)=G(x, b)$ for all $x \in B^{m}$. We also arrange that $b_{1}, \ldots, b_{n}$ are independent over $\mathcal{A}$. We now proceed by a further induction on $n$. If $n=0$ then $g=G$ is $A$-definable, and the desired result holds with $S^{\prime}=S$. In the remainder of the proof we assume that $n>0$. We take a finite partition $\Pi$ of $B^{m+n}$ into cells, all $A$-definable in $\mathcal{B}$, such that if $E \in \Pi$ is open, then either $G \mid E$ is strictly increasing in its last (i.e. $(m+n)$ th) variable, or $G \mid E$ is independent of its last variable, or $G \mid E$ is strictly decreasing in its last variable. For $E \in \Pi$, let $E^{\prime} \subseteq B^{m}$ be the image of $E$ under the projection map $\left(x_{1}, \ldots, x_{m+n}\right) \mapsto\left(x_{1}, \ldots, x_{m}\right): B^{m+n} \rightarrow$ $B^{m}$, and let $S_{E}:=\{x \in S:(x, b) \in E\}$, so that $S_{E} \subseteq E^{\prime}$. Let $E \in \Pi$.

Claim. There exists a set $S_{E}^{\prime} \subseteq S_{E}$ that is definable in $\mathcal{B}$ such that

$$
A^{m} \cap S_{E} \cap g^{-1}(A)=A^{m} \cap S_{E}^{\prime} .
$$

The validity of this claim for every $E \in \Pi$ implies the desired result about $S$ and $g$, because $S$ is the union of the sets $S_{E}$. If $\operatorname{dim}\left(S_{E}\right)<m$, then the claim follows by the inductive assumption. So let $\operatorname{dim}\left(S_{E}\right)=m$. Taking into account that then $E^{\prime}$ is an $A$-definable open cell in $B^{m}$ and that $b_{1}, \ldots, b_{n}$ are independent over $\mathcal{A}$ it follows that $E$ is an open cell.

CASE 1: The function $G \mid E$ is independent of the last variable. Thus there exists a function $G^{\prime}: B^{m+n-1} \rightarrow B$ that is $A$-definable in $\mathcal{B}$ and such that

$$
G^{\prime}\left(x_{1}, \ldots, x_{m+n-1}\right)=G\left(x_{1}, \ldots, x_{m+n-1}, x_{m+n}\right)
$$

for all points $\left(x_{1}, \ldots, x_{m+n-1}, x_{m+n}\right) \in E$. Then we apply the inductive assumption on $n$ to $S_{E}$ in the role of $S$, the function $g^{\prime}: B^{m} \rightarrow B$ given by $g^{\prime}(x)=G^{\prime}\left(x, b_{1}, \ldots, b_{m+n-1}\right)$ in the role of $g$, and the function $G^{\prime}$ in the role of $G$. The claim follows since $g(x)=g^{\prime}(x)$ for $x \in S_{E}$.

CASE 2: The function $G \mid E$ is strictly increasing in the last variable. In that case $A^{m} \cap S_{E} \cap g^{-1}(A)=\emptyset$. To see this, take an $A$-definable function $H: B^{m+n} \rightarrow B$ such that if $x=\left(x_{1}, \ldots, x_{m+n}\right) \in E$ and $G(x)=t$, then $H\left(x_{1}, \ldots, x_{m+n-1}, t\right)=x_{m}$. Suppose $a \in A^{m} \cap S_{E} \cap g^{-1}(A)$. Then $G(a, b)=a^{\prime} \in A$, so that we have $H\left(a, b_{1}, \ldots, b_{n-1}, a^{\prime}\right)=b_{n}$, and thus $b_{n} \in \mathcal{A}\left\langle b_{1}, \ldots, b_{n-1}\right\rangle$, contradicting the independence of $b_{1}, \ldots, b_{n}$ over $\mathcal{A}$.

CASE 3: The function $G \mid E$ is strictly decreasing in the last variable. Then we proceed as in Case 2 .

Lemma 4.3. Let $X \subseteq B$ be $\mathcal{A}$-small. Then $X$ is a finite union of sets of the form $f\left(A^{m} \cap E\right.$ ) (for various $m$ ) where $E$ is an open cell in $B^{m}$ (in the sense of $\mathcal{B}$ ) and $f: E \rightarrow B$ is continuous and definable in $\mathcal{B}$. 
Pr o o f. By definition the set $X$ is definable in $(\mathcal{B}, \mathcal{A})$ and $X \subseteq f\left(A^{m}\right)$ for some function $f: B^{m} \rightarrow B$ that is definable in $\mathcal{B}$. Hence $X=f\left(X^{\prime}\right)$ for some set $X^{\prime} \subseteq A^{m}$ that is definable in $(\mathcal{B}, \mathcal{A})$. By Theorem 2 we have $X^{\prime}=S \cap A^{m}$ for some set $S \subseteq B^{m}$ definable in $\mathcal{B}$, so that $X=f\left(A^{m} \cap S\right)$. We now proceed by induction on $m$. The case $m=0$ is trivial since then either $X=\emptyset$ or $X=\{r\}$ for some $r \in B$. Let $m>0$ and assume the desired result holds for $\mathcal{A}$-small sets $f\left(A^{m} \cap S\right)$ with $f$ and $S$ as above for lower values of $m$. Take a finite partition $\Pi$ of $S$ into cells (in the sense of $\mathcal{B}$ ) such that for each $E \in \Pi$ the restriction $f \mid E: E \rightarrow B$ is continuous. Thus $X$ is the union of the sets $f\left(A^{m} \cap E\right)$ for $E \in \Pi$. The open cells $E \in \Pi$ contribute some of the sets $f\left(A^{m} \cap E\right)$ described in the lemma. Suppose now that $E \in \Pi$ is not open, say $\operatorname{dim}(E)=d<m$. Then there are $i_{1}, \ldots, i_{d}$ with $1 \leq i_{1}<\ldots<i_{d} \leq m$ such that the projection map $\lambda:\left(x_{1}, \ldots, x_{m}\right) \mapsto\left(x_{i_{1}}, \ldots, x_{i_{d}}\right): B^{m} \rightarrow B^{d}$ maps $E$ homeomorphically onto an open cell $E^{\prime}:=\lambda(E)$ in $B^{d}$. Take a map $\mu: B^{d} \rightarrow B^{m}$ that is definable in $\mathcal{B}$ and inverse to $\lambda \mid E$, that is, $\mu(\lambda(x))=x$ for $x \in E$. Then $f\left(A^{m} \cap E\right)=(f \circ \mu)\left(A^{d} \cap E^{\prime} \cap \mu^{-1}\left(A^{m}\right)\right)$. By the previous lemma $A^{d} \cap E^{\prime} \cap \mu^{-1}\left(A^{m}\right)=A^{d} \cap S^{\prime}$ for some set $S^{\prime} \subseteq E^{\prime}$ that is definable in $\mathcal{B}$. Thus by the inductive hypothesis the $\mathcal{A}$-small set $f\left(A^{m} \cap E\right)=(f \circ \mu)\left(A^{d} \cap S^{\prime}\right)$ is of the form required by the lemma.

4.4. Proof of Theorem 4. Let $X \subseteq B$ be $\mathcal{A}$-small and write $X$ as a finite union of sets $f\left(A^{m} \cap E\right)$ as in Lemma 4.3. Since $f$ is continuous the set $f(E)$ is definably connected (in the sense of $\mathcal{B}$ ). Thus, neglecting the trivial case when $f(E)$ is a single point, $f(E)$ is an interval $I$ in $\mathcal{B}$ augmented possibly with one or two endpoints. Since $A^{m} \cap E$ is dense in $E$, the set $f\left(A^{m} \cap E\right) \cap I$ is dense in $I$, and by Lemma 4.1 its complement in $I$ is also dense in $I$. Thus $f\left(A^{m} \cap E\right)$ does have the structure described in Theorem 4 for $\mathcal{A}$-small sets. Using Lemma 4.1 again it follows that $X$ itself has this structure.

Next, let $S \subseteq B$ be definable in $(\mathcal{B}, \mathcal{A})$. By part $(2)$ of Theorem 3 we have $S=\left(S^{\prime} \backslash X\right) \cup Y$ for some set $S^{\prime} \subseteq B$ definable in $\mathcal{B}$, and $\mathcal{A}$-small sets $X$ and $Y$. Thus there exists a partition $-\infty=b_{0}<b_{1}<\ldots<b_{k}<$ $b_{k+1}=+\infty$ of $B$ such that for each $i=0, \ldots, k$ either $S^{\prime} \cap\left(b_{i}, b_{i+1}\right)=\emptyset$, or $\left(b_{i}, b_{i+1}\right) \subseteq S^{\prime}$. If $S^{\prime} \cap\left(b_{i}, b_{i+1}\right)=\emptyset$, then $S \cap\left(b_{i}, b_{i+1}\right)=Y \cap\left(b_{i}, b_{i+1}\right)$ is $\mathcal{A}$-small, and thus has the structure described in Theorem 4 for $\mathcal{A}$-small sets. If $\left(b_{i}, b_{i+1}\right) \subseteq S^{\prime}$, then $S \cap\left(b_{i}, b_{i+1}\right)=\left(b_{i}, b_{i+1}\right) \backslash Z$ for some $\mathcal{A}$-small set $Z$, and we appeal once again to the structure of such sets. (Note that in this argument the initial partition $-\infty=b_{0}<b_{1}<\ldots<b_{k}<b_{k+1}=+\infty$ may have to be refined before we are in the situation described by Theorem 4 for $S$.)

Corollary 4.5. Let $S \subseteq B^{m+n}$ be definable in $(\mathcal{B}, \mathcal{A})$. Then there exists a positive integer $M$ such that whenever $x \in B^{m}$ and $S_{x}$ is finite, then $\left|S_{x}\right|<M$. 
Proof. By induction on $n$ it suffices to prove this for $n=1$. For that case we appeal to a compactness argument and the fact that finiteness of $S_{x}$ is equivalent to the definable condition of $S_{x}$ being discrete in $\mathcal{B}$.

Corollary 4.6. Let $f: B \rightarrow B$ be continuous at all but finitely many points of $B$, and definable in $(\mathcal{B}, \mathcal{A})$. Then $f$ is definable in $\mathcal{B}$.

Pro of. By part (1) of Theorem 3 there is a function $\tilde{f}: B \rightarrow B$ definable in $\mathcal{B}$ and an $\mathcal{A}$-small set $X \subseteq B$ such that $f$ and $\widetilde{f}$ agree outside $X$. Let

$$
-\infty=b_{0}<b_{1}<\ldots<b_{k}<b_{k+1}=+\infty
$$

be such that for each $i \in\{0, \ldots, k\}$ the restrictions of $f$ and $\widetilde{f}$ to $\left(b_{i}, b_{i+1}\right)$ are continuous, and either $X \cap\left(b_{i}, b_{i+1}\right)=\emptyset$ or both $X \cap\left(b_{i}, b_{i+1}\right)$ and $\left(b_{i}, b_{i+1}\right) \backslash X$ are dense in $\left(b_{i}, b_{i+1}\right)$. It follows easily that $f$ and $\widetilde{f}$ agree on each interval $\left(b_{i}, b_{i+1}\right)$, and hence $f$ is definable in $\mathcal{B}$.

The following is an o-minimal analogue of Hrushovski's Lemma 1 in $[2$, $\S 3]$.

LEMmA 4.7. Let $\mathcal{R}$ be an o-minimal expansion of an ordered abelian group with underlying set $R$. Let $\widehat{\mathcal{R}}$ be an $\aleph_{0}$-saturated expansion of $\mathcal{R}$ such that all functions $f: R \rightarrow R$ that are definable in $\widehat{\mathcal{R}}$ are definable in $\mathcal{R}$. Then for all $n$, all sets $S \subseteq R^{n}$ definable in $\widehat{\mathcal{R}}$ are definable in $\mathcal{R}$.

Proof. We proceed by induction on $n$. The hypothesis guarantees that the conclusion holds for $n=1$, and thus $\widehat{\mathcal{R}}$ is o-minimal. Assume inductively that the conclusion holds for a certain value of $n, n>0$. The task of showing that all sets $S \subseteq R^{n+1}$ definable in $\widehat{\mathcal{R}}$ are definable in $\mathcal{R}$ reduces (by cell decomposition and the inductive hypothesis) to verifying the following claim.

Claim. Let $f: R^{n} \rightarrow R$ be definable in $\widehat{\mathcal{R}}$. Then $f$ is definable in $\mathcal{R}$.

This is the case for $n=1$ by hypothesis, so we may assume $n>1$. For each $a \in R$ the function $f_{a}: x \mapsto f(a, x): R^{n-1} \rightarrow R$ is definable in $\widehat{\mathcal{R}}$, and hence in $\mathcal{R}$, so there exists a function $F: R^{N+(n-1)} \rightarrow R$, 0-definable in $\mathcal{R}$, and an element $c \in R^{N}$ such that $f(a, x)=F(c, x)$ for all $x \in R^{n-1}$. Of course, $F$ and $c$ depend here on $a$. Since $\widehat{\mathcal{R}}$ is $\aleph_{0}$-saturated there are finitely many functions $F_{i}: R^{N(i)+(n-1)} \rightarrow R, i=1, \ldots, k$, all 0 -definable in $\mathcal{R}$ such that for each $a \in R$ there is $i \in\{1, \ldots, k\}$ and $c \in R^{N(i)}$ with $f(a, x)=F_{i}(c, x)$ for all $x \in R^{n-1}$. Using dummy variables we can easily combine these functions $F_{1}, \ldots, F_{k}$ into a single function $F: R^{N+(n-1)} \rightarrow R$, definable in $\mathcal{R}$, such that for each $a \in R$ there is $c \in R^{N}$ with $f(a, x)=$ $F(c, x)$ for all $x \in R^{n-1}$. By definability of Skolem functions there is then a map $c=\left(c_{1}, \ldots, c_{N}\right): R \rightarrow R^{N}$, definable in $\widehat{\mathcal{R}}$, such that for all $a \in R$ we have $f(a, x)=F(c(a), x)$ for all $x \in R^{n-1}$. By hypothesis each function $c_{i}$ is definable in $\mathcal{R}$. Thus $f$ is definable in $\mathcal{R}$. 
4.8. Proof of Theorem 5. We consider here $(\mathcal{B}, \mathcal{A})$ as an expansion of the field of real numbers. We have to show that each open subset of $\mathbb{R}^{n}$ that is definable in $(\mathcal{B}, \mathcal{A})$ is already definable in $\mathcal{B}$. For each $L^{2}(\mathbb{R})$-formula $\phi(y)$ with $y=\left(y_{1}, \ldots, y_{n}\right)$ that defines an open subset of $\mathbb{R}^{n}$ in $(\mathcal{B}, \mathcal{A})$ we introduce a new $n$-ary relation symbol $O_{\phi}$. Let $\widehat{L}$ be the language $L$ augmented by these new relation symbols $O_{\phi}$. Let $\widehat{\mathcal{B}}$ be the $\widehat{L}$-expansion of $\mathcal{B}$ in which each new $n$-ary relation symbol $O_{\phi}$ is interpreted as the subset of $\mathbb{R}^{n}$ defined in $(\mathcal{B}, \mathcal{A})$ by $\phi(y)$. By Theorem 4 the open subsets of $\mathbb{R}$ that are definable in $(\mathcal{B}, \mathcal{A})$ are exactly the finite unions of open intervals. It then follows by a result in [5] about expansions of the real field that $\widehat{\mathcal{B}}$ is ominimal, and that a subset of $\mathbb{R}^{n}$ is definable in $\widehat{\mathcal{B}}$ if and only if it is a boolean combination of open subsets of $\mathbb{R}^{n}$ each of which is definable in $(\mathcal{B}, \mathcal{A})$; thus each $\widehat{L}$-formula $\psi(y)$ with $y=\left(y_{1}, \ldots, y_{n}\right)$ is equivalent in $\widehat{\mathcal{B}}$ to a boolean combination of formulas $O_{\phi}(y)$ with $n$-ary $O_{\phi}$ as above. (The structure $\widehat{\mathcal{B}}$ is the open core of $(\mathcal{B}, \mathcal{A})$ in the sense of [5].) Take an $\aleph_{0}$-saturated elementary extension $\left(\mathcal{B}^{*}, \mathcal{A}^{*}\right)$ of $(\mathcal{B}, \mathcal{A})$. We shall apply Lemma 4.7 to $\mathcal{R}:=\mathcal{B}^{*}$, with underlying set $R:=B^{*}$, and its $\widehat{L}$-expansion $\widehat{\mathcal{R}}$ obtained by interpreting each $n$-ary $O_{\phi}$ as above to be the subset of $R^{n}$ defined by the formula $\phi(y)$ in $\left(\mathcal{B}^{*}, \mathcal{A}^{*}\right)$. Clearly, $\widehat{\mathcal{R}}$ is an $\aleph_{0}$-saturated elementary extension of $\widehat{\mathcal{B}}$, and thus also o-minimal. By 4.6 each function $R \rightarrow R$ definable in $\widehat{\mathcal{R}}$ is definable in $\mathcal{R}$. Thus, by 4.7 ,

(*) $\quad \widehat{\mathcal{R}}$ and $\mathcal{R}$ have the same definable subsets of $R^{n}$ for all $n$.

Let now $S \subseteq \mathbb{R}^{n}$ be open and definable in $(\mathcal{B}, \mathcal{A})$. We have to show that $S$ is definable in $\mathcal{B}$. Let $S$ be defined in $(\mathcal{B}, \mathcal{A})$ by the $L^{2}(\mathbb{R})$-formula $\phi(y)$ with $y=\left(y_{1}, \ldots, y_{n}\right)$, and let $S^{*}$ be the subset of $R^{n}=\left(B^{*}\right)^{n}$ defined by this same formula in $\left(\mathcal{B}^{*}, \mathcal{A}^{*}\right)$. By $(*)$ there is an $L$-formula $\phi^{\prime}(x, y)$ with $x=\left(x_{1}, \ldots, x_{m}\right)$ and an element $b^{*} \in\left(B^{*}\right)^{m}$ such that $\phi(y)$ and $\phi^{\prime}\left(b^{*}, y\right)$ are equivalent in $\left(\mathcal{B}^{*}, \mathcal{A}^{*}\right)$. Because $(\mathcal{B}, \mathcal{A})$ is an elementary substructure of $\left(\mathcal{B}^{*}, \mathcal{A}^{*}\right)$ it follows that there exists $b \in \mathbb{R}^{m}$ such that $\phi(y)$ and $\phi^{\prime}(b, y)$ are equivalent in $(\mathcal{B}, \mathcal{A})$. Thus $S$ is defined in $\mathcal{B}$ by the formula $\phi^{\prime}(b, y)$.

In the terminology of [5] we have identified the "open core" of $(\mathcal{B}, \mathcal{A})$ and shown it to be o-minimal. This has some attractive consequences which we list in the following corollary.

Corollary 4.9. Suppose $\mathcal{B}$ expands the ordered field of real numbers. Let $S \subseteq \mathbb{R}^{n}$ be definable in $(\mathcal{B}, \mathcal{A})$. Then:

(1) The closure and interior of $S$ are definable in $\mathcal{B}$.

(2) $S$ is definable in $\mathcal{B}$ if and only if $S$ is a boolean combination of closed subsets of $\mathbb{R}^{n}$ each of which is definable in $(\mathcal{B}, \mathcal{A})$. 
(3) There is a finite partition $\Pi$ of $\mathbb{R}^{n}$ into cells (in the sense of $\mathcal{B}$ ) such that for each $C \in \Pi$ either $C \cap S=\emptyset$, or $C \subseteq S$, or both $C \cap S$ and $C \backslash S$ are dense in $C$.

While (1) and (2) are immediate consequences of the theorem, (3) is proved in [5], where also further refinements are given. Note that (3) extends the second part of Theorem 4 to arbitrary definable sets in the case when $\mathcal{B}$ expands the ordered field of reals.

\section{References}

[1] L. van den Dries and A. Lewenberg, T-convexity and tame extensions, J. Symbolic Logic 60 (1995), 74-102.

[2] E. Hrushovski, Strongly minimal expansions of algebraically closed fields, Israel J. Math. 79 (1992), 129-151.

[3] A. Macintyre, Dense embeddings I: a theorem of Robinson in a general setting, in: Model Theory and Algebra. A Memorial Tribute to Abraham Robinson; D. H. Saracino and V. B. Weispfenning (eds.), Lecture Notes in Math. 498, Springer, Berlin, $1975,200-219$.

[4] H. D. MacPherson, D. Marker and C. Steinhorn, Weakly o-minimal theories and real closed fields, preprint.

[5] C. Miller and P. Speissegger, Expansions of the real line by open sets: o-minimality and open cores, preprint.

[6] Y. Peterzil and S. Starchenko, A trichotomy theorem for o-minimal structures, Proc. London Math. Soc., to appear.

[7] A. Pillay and C. Steinhorn, Definable sets in ordered structures. I, Trans. Amer. Math. Soc. 295 (1986), 565-592.

[8] A. Robinson, Solution of a problem of Tarski, Fund. Math. 47 (1959), 79-204.

Department of Mathematics

University of Illinois

Urbana, Illinois 61801

U.S.A.

E-mail: vddries@math.uiuc.edu

Received 24 July 1997;

in revised form 30 December 1997 